\title{
Frequency of seafood intake in pregnancy as a determinant of birth weight: evidence for a dose dependent relationship
}

\author{
Sjúrdur F Olsen, Philippe Grandjean, Pál Weihe, Tórbjørn Viderø
}

\begin{abstract}
Study objective-The aim was to explore whether maternal consumption of seafood is a determinant of birth weight in a dose dependent manner.

Design-A population based survey of lifestyle factors in pregnancy was linked with information from antenatal and obstetric records.

Setting and participants-Seventy five per cent of all 1362 women who delivered in the Faroe Islands during the study period 198687 who gave a structured post partum interview on lifestyle factors.

Main results-Altogether, 2, 6, 16, 33, 26, 14, and $3 \%$ of women had consumed approximately $0,1,2,3,4,5$, and $6+$ respectively seafood (fish or whale) dinner meals per week during pregnancy. The average birth weight $(p=0.02)$ and birth length $(p=0.002)$ varied significantly between the seven groups, and increased by about $0.2 \mathrm{~kg}$ and $1 \mathrm{~cm}$, respectively between women who ate 0 and those who consumed 3 seafood meals per week. Mean birth weight and length tended to level off with further fish consumption: when fitting a second degree polynomial, the quadratic terms were negative and significant for both birth weight $(p=0.005)$ and length $(p=0 \cdot 001)$. Analogous analyses for pregnancy duration were not significant, but exhibited similar trends. All analyses were adjusted for maternal height, weight, parity, age, marital status, and smoking.

Conclusions-The weight and length of the newborn increased with the frequency of seafood dinner meals consumed in pregnancy but only up to a consumption level of about 3 meals per week.
\end{abstract}

f Epidemiol Community Health 1993; 47: 436-440

Eating seafood, which is rich in long chain n-3 fatty acids, may increase birth weight in two ways: it may prolong the duration of pregnancy by interfering with the prostaglandins involved in the process of parturition ${ }^{1}$ and it may increase the fetal growth rate by raising the ratio of biologically active prostacylins to thromboxanes and reducing blood viscosity, thereby facilitating placental blood flow. ${ }^{2-4}$ It has been suggested ${ }^{5}$ that the effect of n-3 fatty acids on pregnancy duration may depend on the level of consumption. A direct correlation was seen between n-3 fatty acids quantified in maternal erythrocytes and pregnancy duration in a sample of Danish women, whereas no such association could be detected in a sample of Faroese women who tended to eat more fish. This finding suggested the existence of a saturation level for the effect of n-3 fatty acids on pregnancy duration, and that a substantial proportion of women in the Faroe Islands may have an intake that is above this level.

We studied the relationships between the size of the newborn and pregnancy duration on the one hand, and self reported consumption of seafood dinner meals on the other, in a larger sample of Faroese women ${ }^{67}$ to investigate whether the relationships do depend on the level of consumption. We also estimated to what extent the higher consumption of seafood in the Faroes might explain the difference in the birth weight between the Faroes and Denmark.

\section{Population and methods}

DATA COLLECTION

Data were collected for all liveborn singleton deliveries in the Faroes between March 1, 1986 and December 31, 1987 (with a break from July 10 to August 8,1987 ). The cohort has been described elsewhere. ${ }^{6}{ }^{7}$ After delivery, the midwife asked the mother standardised questions about fish (number of fish dinner meals per week) and pilot whale (number of whale dinner meals per month) consumption and smoking habits $(0,1-10$, or $11+$ cigarettes per day) on average during pregnancy. The original data set (on the basis of which some preliminary analyses of birth weight were undertaken ${ }^{7}$ ) was supplemented further with information about maternal height and weight, placental weight, and with all available information for gestational age assessment; this information was extracted from birth certificates and antenatal records from midwife assessments and ultrasound examinations. A computer file of all routine parameters for all deliveries in the Faroes during the study period was also obtained from the Birth Registry at the National Board of Health. Altogether $77 \%$ of all eligible deliveries came from the uptake area of the Central Hospital in Tórshavn, the remainder came from the uptake areas of the other two hospitals in the Faroes (Klaksvik and Tvøroyi).

\section{DERIVED VARIABLES}

Gestational age estimation was based primarily on the date of the last menstrual period. When the date was uncertain, the cycle irregular or prolonged (>35 days), or the contraceptive pill had been used up to four months before conception, the gestational age was based on ultrasound assessment of the biparietal diameter. If that was not available either, the best clinical judgement recorded by the midwife on the birth certificate was used. 
"Number of seafood dinners per week" was obtained by adding the number of fish dinners recorded per week to the number of pilot whale dinners recorded per month, after dividing the latter by four and rounding it up to the nearest integer. The two variables could be combined in this way since we estimated the n-3 fatty acid content in a typical pilot whale meal to be $0.5-1.0 \mathrm{~g}$ per $100 \mathrm{~g}$ meat+blubber (based on personal information from Dr Petur Zachariassen, The Faroese Academy (dietary survey in the Faroes 1980-81), and Dr Gudmundur Haraldsson, University of Iceland (fatty acid analysis of meat and blubber)), which is within the range of the $n-3$ fatty acid content in the fish species commonly eaten in the Faroes. Furthermore, stratified analyses undertaken of each of the two variables, while keeping the other variable constant (with a consequential substantial loss of statistical power), suggested that the pregnancy outcome was related to the two types of seafood dinners in fairly similar ways; in particular, that there was a tendency to level off at high levels of both dinner variables (data not shown). The combined variable defined seven categories of women consuming approximately $0,1,2,3,4,5$, and $6+$ seafood dinners per week.

STATISTICAL ANALYSIS

Analyses of variance were applied to compare the seven nutritional groups in respect of mean gestational age, birth weight, birth length, and placental weight. Multiple classification analyses? were applied to adjust these comparisons for differences between the groups in maternal height, weight, parity, age, marital status, and smoking and to adjust further the comparisons of mean birth weight, mean birth length, and mean placental weight for differences in gestational age. These statistical models have the advantage of being free of any assumptions about the shape of the relationship between the seafood dinners variable and the pregnancy outcome variable.

Linear trends were tested for by linear regression. Levelling off of the relationships, suggested by an earlier study, ${ }^{5}$ was tested for by fitting the data to a second degree polynomial $\left(b_{0}+b_{1} x+b_{2} x^{2}\right)$ in a regression model. These analyses were also adjusted for the potential confounders listed above, by including them in the model as explanatory variables.

Table I Gestational age, birth weight, birth legnth, and placental weight in relation to frequency of maternal consumption of seafood dinners in Faroese women. The $p$ values refer to the tests of the variability of the means between the seven nutritional groups, after adjustment for confounding by maternal height, weight, parity, age, marital status, and smoking (multiple classification analysis). For each nutritional group the fitted, smoking (multiple classification analysi
confounder adjusted vales are shown

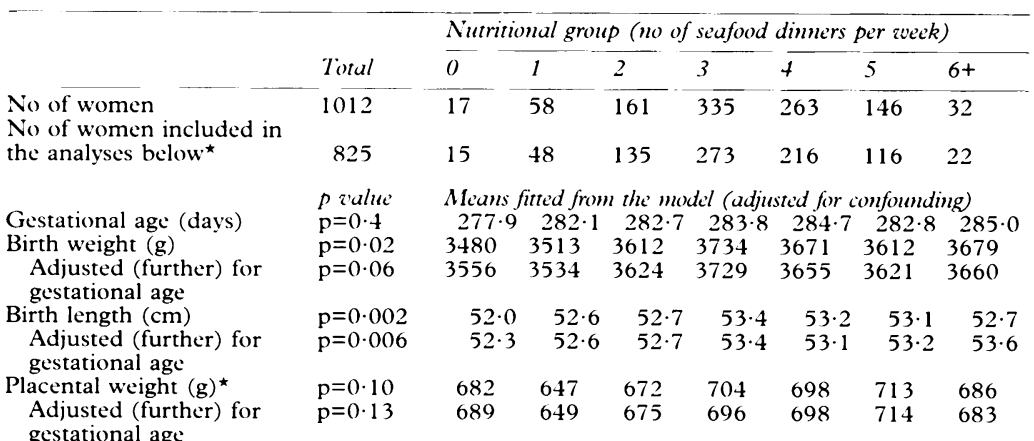

Numbers included in the analyses were smaller because of missing values for covariates; in case of placental weight, total no was 767
Birth weight tended to be related linearly to maternal height, weight before pregnancy, parity, and age, when they were categorised as: -150 , $151-160,161-170$, and $171+\mathrm{cm} ;-45,45-50$, $51-70$, and $71+\mathrm{kg}$; para $0,1-2$, and $3+;-19$, $20-34$, and $35+$ years respectively. These categories were coded $0,1,2, \ldots$ in the multivariate analyses since the analyses assume linear relationships between the pregnancy outcome variable and the confounding variables.

The term significant denotes a two sided $\mathrm{p}$ level of 0.05 or less.

\section{Results}

The sample comprised $75 \%$ of all deliveries that took place during the study period. Deliveries not included in the study had a significantly shorter mean gestational age than those included, whereas no significant differences were seen in the average maternal age, parity, height, weight before pregnancy, birth length, or placental weight. ${ }^{6}$

The women included in the study had an mean (SD) age, height, and weight before pregnancy of $26.9(5.53)$ years, $164.0(5.64) \mathrm{cm}$, and 60.1 (9.54) $\mathrm{kg}$ respectively, and $35 \%, 62 \%$, and $63 \%$ respectively were primiparous, married, and had reported not to have smoked in pregnancy. Mean (SD) gestational age, birth weight, birth length, and placental weight were $283.4(12.6)$ days, $3669(543) \mathrm{g}, 53 \cdot 1(2 \cdot 28) \mathrm{cm}$, and $695 \cdot 1(149) \mathrm{g}$ respectively. The distribution of the women according to the seafood variable is given in table I.

For all four pregnancy outcomes, the fitted, confounder adjusted values from the multiple classification analyses (table I) exhibited patterns that were similar to those seen in the unadjusted comparisons of the group means (figure). In the adjusted analyses (table I), mean birth weight and length varied significantly between the seven nutritional groups; however, mean gestational age and placental weight did not. Further adjustment for gestational age at birth reduced the variation in mean birth weight, birth length, and placental weight somewhat, although substantial significant variations remained, particularly for birth weight and length (table I).

Gestational age, birth weight, and birth length tended to increase with the number of seafood dinners (figure and table I), but the rise was apparent only up to a consumption level corresponding with the group in the middle of the distribution, above which the curves tended to level off. Placental weight seemed to fluctuate somewhat more, but it tended to rise over the total range of exposure.

When applying linear regression, only placental weight increased significantly $(p=0.02)$ over the total range of the seven groups (table II, linear model). This association was rather stable towards adjusting for birth weight by including it as a predictor variable in the linear model.

When fitting a second degree polynomial $\left(b_{0}+b_{1} x+b_{2} x^{2}\right)$, the coefficients of the quadratic terms were negative and highly significant for both birth weight $(p=0.005)$ and length $(p=0.001)$ (table II, polynomial model). For gestational age $(p=0 \cdot 1)$ and placental weight $(p=0 \cdot 3)$, they were not significant. 
Unadjusted means (SEM) of gestational age, birth weight, birth length, and placental weight in relation to the frequency of seafood dinner meals consumed during pregnancy by mothers living in the Faroe Islands $(n=1012$, for placental weight $n=917$ )

Table II Fitting a line and a second degree polynomial to the data. In the linear model the seafood dinners variable (coded as 0, 1, 2, 3, 4, 5, 6) is included as a linear term $(x)$, whereas in the sencond degree polynomial model it is included as a linear plus a quadratic term $\left(x+x^{2}\right)$. Shown are the regression coefficients (95\% confidence intervals, $p$ value) for the linear and the quadratic terms. The regression coefficients were adjusted for potential confounding by maternal height, weight, parity, age, marital status, and smoking *
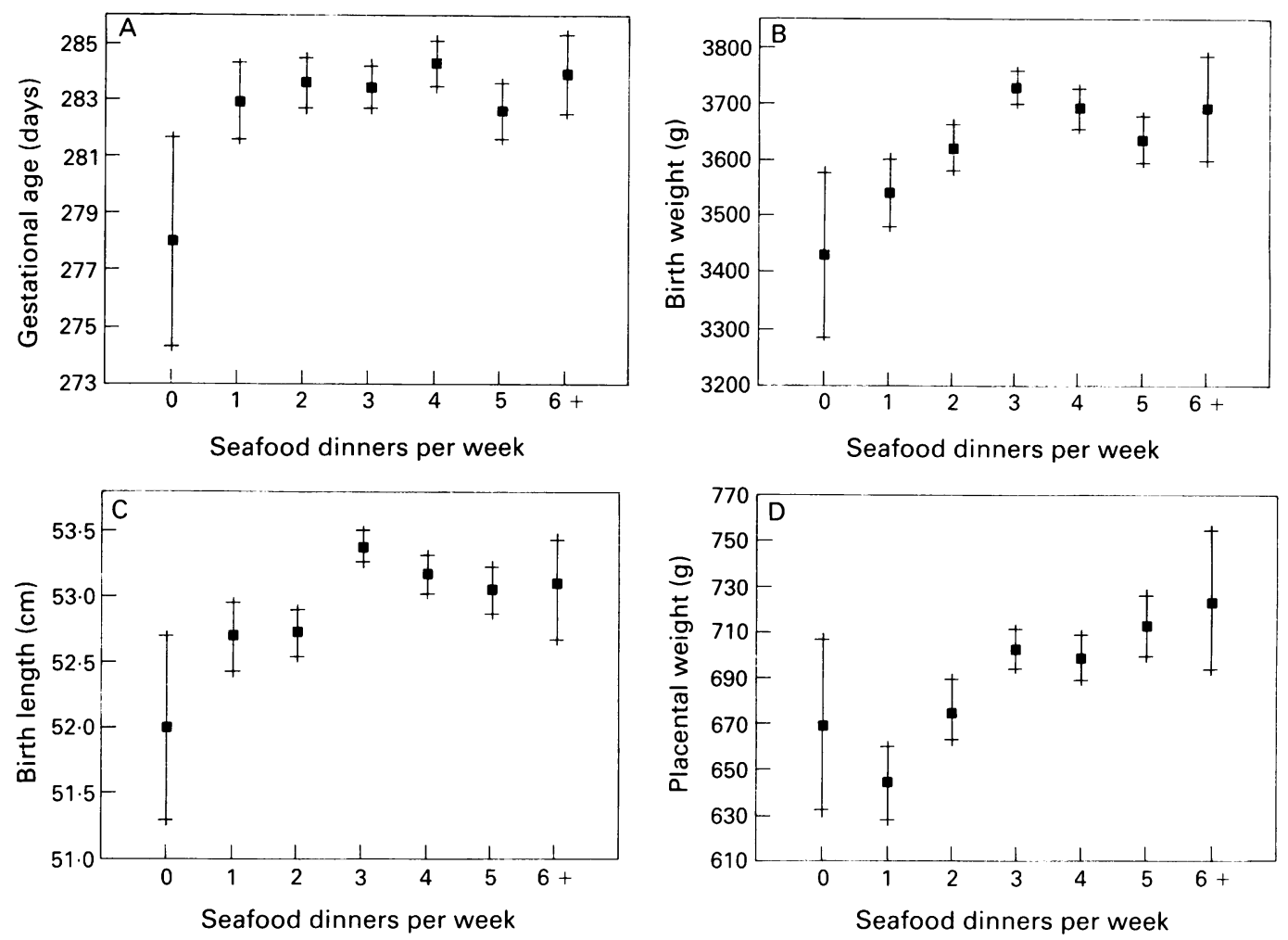

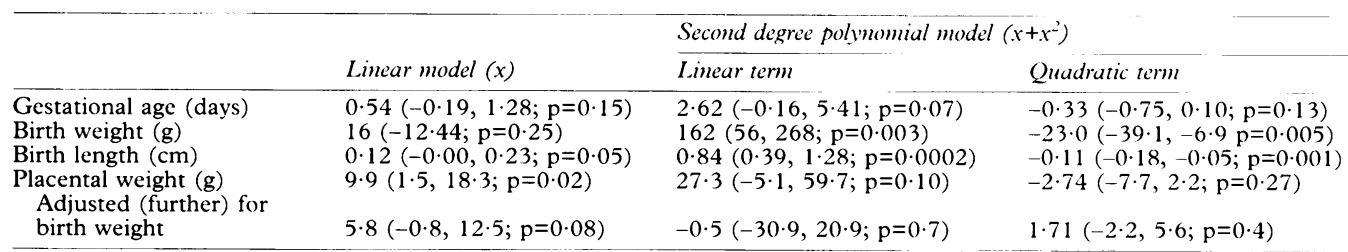

${ }^{\star}$ For gestational age, birth weight and birth length, $\mathrm{n}$ was 825 ; for placental weight, $\mathrm{n}$ was 767

\section{Discussion}

We found that birth weight and length varied significantly and substantially across the seven nutritional groups. However, the relationship seemed not to be of a simple, linear type. Thus, increases were seen of about $200 \mathrm{~g}$ in birth weight and $1 \mathrm{~cm}$ in birth length from the group consuming no seafood and up to a consumption level of about 3 seafood dinners per week; above that level, no further increases were seen. This shape of the relationships could not be explained by random fluctuation since, in case of birth weight and length, fitting a second degree polynomial resulted in a negative and highly significant coefficient for the quadratic term. The corresponding analyses for pregnancy duration were not significant, although pregnancy duration tended to increase with maternal intake of seafood in a similar, dose dependent manner.

Several explanations for these results may be considered, including various types of biases. Only $75 \%$ of eligible women were included in the study, and potential selection bias may be of importance. However, restricting the analyses to women living in the uptake area of the Central Hospital in Tórshavn where as many as $85 \%$ of all eligible deliveries that took place during the study period were included in the study sample, resulted in conclusions similar to those described (data not shown), making this explanation less likely. Going from bivariate to multivariate analyses reduced the study sample by $18 \%$ because of missing values for covariates (table I); however, bivariate analyses undertaken in the full (figure) and the reduced sample (data not shown) exhibited very similar patterns.

Lifestyle data were collected after delivery which could theoretically lead to recall bias, but it is unlikely that the pregnancy outcomes dealt with here would affect the information collected on seafood intake.

The crude level of exposure classification must also be kept in mind. Thus, the exposure variable is frequency of seafood dinner meals, and not quantified exposure to a specific dietary factor. The variable is likely to correlate with the intake of a number of dietary components of seafood, including fat soluble vitamins, selenium, and environmental pollutants. Seafood is a traditional diet in the Faroe Islands, and the comparisons may be confounded by factors reflecting the genetic setting or the traditional lifestyle of the Faroese population; however, adjusting for confounding by mother's smoking, height, weight, parity, age, and marital status did not change the estimates to any substantial degree. We have no data on how the nutritional groups compare as to socioeceonomic status. In our judgement, increased consumption of fish and pilot whale tends, if anything, to be associated inversely with socioeconomic status in this particular population. 
Nevertheless, one of our aims was to use the data set to test the hypothesis that $\mathrm{n}-3$ fatty acids from marine food sources may increase birth weight. ${ }^{1}$ The rationale is that seafood is by far the most important food source of long chain n-3 fatty acids. From this point of view, and with the above limitations in mind, we may at least conclude that our test does not reject the possibility that n-3 fatty acids ingested in pregnancy are a determinant of birth weight. Pregnancy duration showed the same trend as birth weight but the variation across the nutritional groups was not statistically significant, and the associations seen with birth weight were rather stable towards adjustment for gestational age. Because of the inherent imprecision of the gestational age assessment, however, these findings should be interpreted with caution in respect of the question whether the postulated effect of birth weight is mediated by a prolongation of the intrauterine growth period ${ }^{1}$ or an increase in the fetal growth rate, ${ }^{2-4}$ but the data are more in accordance with the latter than the first possibility. Placental weight measured in routine clinical practice is likely to be an even more imprecise measure, because comparatively little attention is attached to it. It is therfore a noteworthy finding that placental weight tended to rise in relation to seafood dinners over the total range of exposure, and also that this association seemed to be mediated only partly by the association with birth weight (adjusting for birth weight reduced the point estimate of the regression coefficient for placental weight from 9.9 to $5 \cdot 8$, table II, linear model). Indeed, this finding could reflect the postulated effect of $n-3$ fatty acids on placental blood flow. ${ }^{2-4}$

On the other hand, a simple linear relationship between the frequency of seafood meals and birth weight was not in accordance with the data. These suggested a dose dependent relationship instead. This possibility was forseen before the analyses as an a priori hypothesis on the basis of two earlier studies. One was a study of the relationship between gestational age and marine n- 3 fatty acids measured in erythrocyte lipids, ${ }^{5}$ where the relationships tended to be steeper in a population with a low level of exposure than in one with high level of exposure. The other study was a controlled trial, ${ }^{9}$ showing a prolonged gestation and a correspondingly increased birth weight in Danish women after supplementation with fish oil from week 30 of gestation, except that no effect could be detected in the subgroup of women with comparatively high fish consumption at entry to the trial. The present study provides further support to the possibility of a saturable, dose dependent relationship between n-3 fatty acids and weight at birth. Again, various sources of bias could possibly account for the bending of the curves. Thus, the nutritional variable is likely to have a large misclassification error when used as a surrogate measure for the intake of marine n-3 fatty acids, and it is possible that misclassification is greatest in the upper range of the scale (for example, it may be easier to be sure about whether one has consumed a particular diet zero, one, or two times than whether one has consumed it four, five, six times a week); if so, this would tend to flatten artificially the upper part of a possible linear relationship.
There is a considerable, unexplained variation between the Nordic populations in average birth weight. ${ }^{10}$ The difference between the highest and lowest populations, the Faroese and the Danish, is about 200 g. ${ }^{11}$ In Denmark, $16 \%, 75 \%$, and $9 \%$ of the pregnant women have been found to consume $0,1-4$, and 5 or more fish dinner meals per month, respectively. ${ }^{3}$ Using this distribution to calculate a weighted average birth weight for the Faroes from the corresponding confounder adjusted fitted values from the multiple classification analyses $(3840 \mathrm{~g}, 3513 \mathrm{~g}$, and $3612 \mathrm{~g}$ for the groups consuming 0,1 , and 2 seafood dinner meals per week, respectively (table I)), resulted in an overall average birth weight of $3517 \mathrm{~g}$. This may be taken as a crude approximation for the expected birth weight average, predicted from the model, had the Faroese women had a similar level of seafood dinner consumption as the Danish women. The figure is about $150 \mathrm{~g}$ lower than the average actually observed in the Faroese sample ( $3669 \mathrm{~g}$ ). Differences between the Faroes and Denmark in the frequency of seafood dinners consumed in pregnancy thus seem to account for a substantial part of the difference in average birth weight observed $\left(200 \mathrm{~g}^{11}\right)$ between the two populations.

A birth weight difference of a similar magnitude has recently been described between the Orkneys and an area in the Scottish mainland. ${ }^{12}$ The authors concluded from a multiple regression analysis that the difference could largely be ascribed to differences in known determinants of birth weight, particularly pregnancy duration, maternal smoking, height, and weight, rather than the higher fish consumption observed among the islanders. After having adjusted for the factors mentioned, however, the Orkney residents still had a $62 \mathrm{~g}$ higher average birth weight compared with the mainland residents. No corresponding adjusted analysis was presented for the differences observed between the areas in gestational age, which was 0.36 weeks. Despite the title of the paper, ${ }^{12}$ these data do not reject any of the two possibilities that dietary marine $n-3$ fatty acids may prolong duration of pregnancy and increase fetal growth rate.

Despite its limitations we think that the present study does provide evidence in support of the hypothesis that marine food products ingested in pregnancy increase the baby's size at birth, and also that the dose-response relationship has a saturation level. Both conclusions are of potential importance for public health. Further studies are needed which make use of more specific and quantitative measures for the $n-3$ fatty acid intake, which are better able to disentangle and isolate any effects on pregnancy duration from those on fetal growth, and which focus on endpoints of greater importance to public health like occurrences of preterm delivery and intrauterine growth retardation.

The study was supported financially by the Danish Medical Research Council, the Danish Health Foundation, the Højgaard Foundation, the Vestnorden Foundation, the Danish Agency for Environmental Protection, the Director Jacob Madsens Foundation, and Michaelesen Fonden.

We are indebted to Professor J Olsen, Professor N J Secher, and Professor T I A Sorensen and to Associate Professor, Statistician M Væth for valuable comments 
on the manuscript and analyses, and to Ms Minna Jacobsen for her meticulous handling of data from birth certificates and antenatal records. The National Board of Health kindly provided a file from the Medical Birth Register.

1 Olsen SF, Hansen HS, Sørensen TIA, Jensen B, Secher NJ Sommer $S$, et al. Intake of marine fat, rich in $n-3$ polvunsaturated fatty acids, may increase birthweight by polyunsaturated fatty acids, may increase birth
prolonging gestation. Lancet 1986; ii: 367-9.

2 Andersen HJ, Andersen LF, Fuchs AR. Diet, pre-eclampsia and intrauterine growth retardation. Lancet 1989; i: 1146. and intrauterine growth retardation. Lancet 1989; i: 1146 .
Olsen SF, Olsen J, Frische G. Does fish consumption during Olsen SF, Olsen J, Frische G. Does fish consumption during
pregnancy increase fetal growth? A study of the size of the newborn, placental weight and gestational age in relation to fish consumption during pregnancy. Int $\mathcal{f}$ Epidemiol 1990 19: $971-7$.

4 Secher NJ, Olsen SF. Fish oil and pre-eclampsia. Br $\mathcal{f O}$ () bstet Gynaecol 1990; 67: 1077-9.

5 Olsen SF, Hansen HS, Sommer S, Jensen B, Sorensen TIA Secher NJ, Zachariassen P. Gestational age in relation to marine n-3 fatty acids in maternal erythrocytes: a study of women in the Faroe Islands and Denmark. Am $f$ Obstet Gynecol 1991; 164: 1203-9.
6 Grandjean P, Weithe P, Jorgensen PJ, Clarkson T, Cernichiari E, Viderø T. Impact of maternal seafood diet on Cernichiari E, Videro T. Impact of maternal seafood dict on fetal exposure to mercury,

7 Grandjean P, Weithe P. Neurobehavioral effects of intrauterine mercury exposure: potential sources of bias. Environ Re's 1993

8 SPSS Inc. SPSSPC+ I'2.0 Base Manual for the IBMI $P C / X T / A T$ and PS/2. Chicago: SPSS Inc, 1988: B174 $\mathrm{B} 175$ and $\mathrm{C} 20-\mathrm{C} 21$

9 Olsen SF, Sorensen JD, Secher NJ, et al. Randomised controlled trial of effect of fish-oil supplementation on pregnancy duration. Lancet 1992; 339: 1003-7.

10 NOMESKO. Births and infant mortality in the Nordic countrics. Copenhagen: NOMESKO, 1993. NOMESKO Publication no 39.

11 Olsen SF, Joensen HD. High liveborn birthweights in the Faroes: a comparison between birthweights in the Faroes and in Denmark f Epidemiol Community Health 1985; 39 . $27-32$.

12 Harper V, MacInnes R, Campbell D, Hall M. Increased birth weight in northerly islands: is fish consumption a red herring? BMF 1991; 303: 166 